\title{
Lattice study of meson correlators in the $\epsilon$-regime of two-flavor QCD
}

\author{
H. Fukaya, ${ }^{1}$ S. Aoki, ${ }^{2,3}$ S. Hashimoto, ${ }^{4,5}$ T. Kaneko, ${ }^{4,5}$ H. Matsufuru, ${ }^{4}$ J. Noaki, ${ }^{4}$ K. Ogawa, ${ }^{6}$ T. Onogi, ${ }^{7}$ and N. Yamada ${ }^{4,5}$
}

(JLQCD collaboration)

\author{
${ }^{1}$ The Niels Bohr Institute, The Niels Bohr International Academy, Blegdamsvej 17 DK-2100 Copenhagen Ø Denmark \\ ${ }^{2}$ Graduate School of Pure and Applied Sciences, University of Tsukuba, Tsukuba 305-8571, Japan \\ ${ }^{3}$ Riken BNL Research Center, Brookhaven National Laboratory, Upton, New York 11973, USA \\ ${ }^{4}$ High Energy Accelerator Research Organization (KEK), Tsukuba 305-0801, Japan \\ ${ }^{5}$ School of High Energy Accelerator Science, The Graduate University for Advanced Studies (Sokendai), Tsukuba 305-0801, Japan \\ ${ }^{6}$ Physics Department, Center for Theoretical Sciences, \\ and National Center for Theoretical Sciences, National Taiwan University, Taipei 10617, Taiwan \\ ${ }^{7}$ Yukawa Institute for Theoretical Physics, Kyoto University, Kyoto 606-8502, Japan
}

(Received 12 December 2007; published 3 April 2008)

\begin{abstract}
We calculate mesonic two-point functions in the $\epsilon$-regime of two-flavor QCD on the lattice with exact chiral symmetry. We use gauge configurations of size $16^{3} \times 32$ at $a \sim 0.11 \mathrm{fm}$ generated with dynamical overlap fermions. The sea quark mass is fixed at around $3 \mathrm{MeV}$ and the valence quark mass is varied in the range $1-4 \mathrm{MeV}$, both of which are in the $\epsilon$-regime. We find a good consistency with the expectations from the next-to-leading order calculation in the $\epsilon$-expansion of (partially quenched) chiral perturbation theory. From a fit we obtain the pion decay constant $F=87.3(5.6) \mathrm{MeV}$ and the chiral condensate $\Sigma^{\overline{\mathrm{MS}}}=$ $[239.8(4.0) \mathrm{MeV}]^{3}$ up to next-to-next-to-leading order contributions.
\end{abstract}

DOI: 10.1103/PhysRevD.77.074503

PACS numbers: $12.38 . G c$

\section{INTRODUCTION}

In the low-energy limit, the dynamics of quantum chromodynamics (QCD) is dominated by the pion fields that appear as pseudo-Nambu-Goldstone bosons associated with the spontaneous breaking of chiral symmetry. Although chiral perturbation theory (ChPT) is a powerful effective theory in understanding their interactions, it has many parameters, the so-called "low-energy constants (LECs)," which should be determined either from experimental data or preferably from direct calculations based on the underlying theory, i.e. QCD. The leading order of ChPT is completely controlled by the two LECs, the chiral condensate $\Sigma$, and the pion decay constant $F$, while at higher orders there are increasing number of LECs.

Numerical simulation of lattice QCD offers the most promising approach to achieve the direct calculation of LECs. In fact, the recent advances in the simulation techniques allow us to calculate, for instance, the pion decay constant to a remarkable precision. However, such calculations may contain nontrivial systematic effects, since the infinite volume limit must be taken before the chiral limit is approached. The violation of chiral symmetry of lattice fermions also seriously complicates the analysis, because ChPT itself must be modified to incorporate the explicit breaking of chiral symmetry [1].

Recently, an alternative approach has been proposed, that is the lattice calculation in the $\epsilon$-regime of ChPT [2-7]. In this approach, the lattice simulation is performed near the chiral limit at a fixed volume $V$. Finite volume effect becomes prominent due to the long-distance corre- lation of the pion fields, which can be treated in a systematic way within ChPT. Of particular interest is the region where the pion correlation length, or the inverse pion mass $1 / m_{\pi}$, exceeds the size of the box $L$

$$
\frac{1}{\Lambda_{\mathrm{QCD}}} \ll L \ll \frac{1}{m_{\pi}}
$$

with $\Lambda_{\mathrm{QCD}}$ the QCD scale. In this $\epsilon$-regime, the zeromomentum mode of the pion fields has to be treated nonperturbatively, and the contribution from nonzero momentum modes is expanded in a new parameter $\epsilon$ :

$$
\frac{m_{\pi}}{\Lambda_{\mathrm{cut}}} \sim \frac{p^{2}}{\Lambda_{\mathrm{cut}}^{2}} \sim \epsilon^{2},
$$

where $p$ denotes pion momentum and $\Lambda_{\text {cut }}$ is a cutoff of ChPT. With this expansion, the volume and topological charge dependence of the chiral condensate, meson correlators, etc., can be written with the same low-energy constants as in the infinite volume.

Simulating lattice QCD in the $\epsilon$-regime has several advantages over the conventional approach. First of all, the infinite volume limit is not necessary when approaching the chiral regime. In the $p$-regime, on the other hand, it is not clear at which masses one can safely apply the chiral expansion to extrapolate lattice data to the physical up and down quark masses. This is a question that depends on the quantity of interest, and therefore one potentially needs to work on a fairly large volume lattice to ensure the condition $m_{\pi} L \gg 1$. In the $\epsilon$-regime, pion mass is made arbitrarily small at a finite volume. The $\epsilon$-expansion requires 
$4 \pi F L \gg 1$, which does not strongly depend on the quark mass.

One may wonder that the computational cost to simulate arbitrarily light sea quark could be prohibitively high, but it is not the case because the lowest-lying quark eigenvalue stays finite at the order of $1 / \Sigma V$ for a given volume $V$ (except for the exact zero modes). In the $\epsilon$-regime the lowest eigenvalue is even lifted by about a factor of $N_{f}$, the number of flavors, according to the chiral random matrix theory.

Another major advantage of the lattice calculation in the $\epsilon$-regime is the prominent dependence of physical observables on the topological charge and sea quark mass. The best known example is the analytic predictions for the lowest-lying eigenvalues derived from the chiral random matrix theory. Utilizing these, precise determination of the low-energy constants has been attempted through direct calculations of the low-lying Dirac eigenvalues mainly in quenched QCD [8-10]. Another possibility is to study hadron correlators in the $\epsilon$-regime, which has also been carried out by several groups in quenched QCD [11-16]. We extend these works to unquenched QCD in this paper. However, this is not an easy task since the exact chiral symmetry is essential in the $\epsilon$-regime. We therefore use the overlap-Dirac operator $[17,18]$ which realizes the exact chiral symmetry on the lattice $[19,20]$. The overlap fermion is much more difficult to simulate than other lattice fermion formulations for both algorithmic and computational reasons.

The recent series of work by the JLQCD Collaboration [21-23] has opened a new possibility of simulating unquenched QCD in the $\epsilon$-regime (see also [24-31] for other exploratory studies). We performed two-flavor dynamical overlap fermion simulations with the quark mass near the chiral limit, on a $16^{3} \times 32$ lattice at a lattice spacing $a \sim$ $0.11 \mathrm{fm}$ (determined with $r_{0} \sim 0.49 \mathrm{fm}$ [32] as an input). We use the Iwasaki gauge action $[33,34]$ with extra Wilson fermions and ghosts to fix the topological charge [35-37]. The sea quark mass $m$ is around $3 \mathrm{MeV}$, which is well within the $\epsilon$-regime. Since the eigenvalue of the Hermitian overlap-Dirac operator is bounded from below, numerical simulation is stable even with such a small quark mass. Comparing the Dirac spectrum with the predictions of the chiral random matrix theory, we extracted the value of the chiral condensate at the leading order of the $\epsilon$-expansion as $\Sigma^{\overline{\mathrm{MS}}}=[251(7)(11) \mathrm{MeV}]^{3}$, where the second error is an estimate of the systematic error due to the next-to-leading order (NLO) effects in the $\epsilon$-expansion.

In this paper, we use the same set of gauge configurations in the $\epsilon$-regime to calculate the meson correlators in various channels. The analytic predictions of ChPT for the pseudoscalar, scalar, axial-vector, and vector channels are known to NLO in the $\epsilon$-expansion [38,39], which are recently extended to the partially quenched ChPT [4042]. We use these ChPT predictions to extract $\Sigma$ and $F$ at the NLO accuracy.

This paper is organized as follows. In Sec. II we review the (partially quenched) ChPT predictions for the meson correlators. The setup of the numerical simulations is given in Sec. III. In Sec. IV, we measure the axial-vector and pseudoscalar correlators to extract $F$ and $\Sigma$. Then, some consistency checks are done using other channels and partially quenched correlators. Comparison of the result for $\Sigma$ is also made with that from the Dirac eigenvalue spectrum. Our conclusions are given in Sec. VI.

\section{II. (PARTIALLY QUENCHED) CHIRAL PERTURBATION THEORY IN THE $\epsilon$-REGIME AT FIXED TOPOLOGY}

In this section, we briefly review the results for the meson correlators calculated within (partially quenched) ChPT. For the full details we refer the original papers $[38,39,41]$. Here we consider $N_{v}$ valence quarks with a mass $m_{v}$ and $N_{f}=2$ degenerate sea quarks with a mass $m_{s}$, both in the $\epsilon$-regime.

As a fundamental building block for the later use, let us define the partially quenched zero-mode partition function $[43,44]$ at a fixed topological charge $\nu$. In addition to the $N_{f}=2$ physical quarks (of mass $m_{s}$ ), a pair of a valence quark (of mass $m_{v}$ ) and a bosonic quark (of mass $m_{b}$ ) is introduced for the partial quenching;

$$
Z_{\nu}^{\mathrm{PQ}}\left(\mu_{b} \mid \mu_{v}, \mu_{s}\right) \equiv \frac{1}{\left(\mu_{s}^{2}-\mu_{v}^{2}\right)^{2}} \operatorname{det}\left(\begin{array}{cccc}
K_{\nu}\left(\mu_{b}\right) & I_{\nu}\left(\mu_{v}\right) & I_{\nu}\left(\mu_{s}\right) & I_{\nu-1}\left(\mu_{s}\right) / \mu_{s} \\
-\mu_{b} K_{\nu+1}\left(\mu_{b}\right) & \mu_{v} I_{\nu+1}\left(\mu_{v}\right) & \mu_{s} I_{\nu+1}\left(\mu_{s}\right) & I_{\nu}\left(\mu_{s}\right) \\
\mu_{b}^{2} K_{\nu+2}\left(\mu_{b}\right) & \mu_{v}^{2} I_{\nu+2}\left(\mu_{v}\right) & \mu_{s}^{2} I_{\nu+2}\left(\mu_{s}\right) & \mu_{s} I_{\nu+1}\left(\mu_{s}\right) \\
-\mu_{b}^{3} K_{\nu+3}\left(\mu_{b}\right) & \mu_{v}^{3} I_{\nu+3}\left(\mu_{v}\right) & \mu_{s}^{3} I_{\nu+3}\left(\mu_{s}\right) & \mu_{s}^{2} I_{\nu+2}\left(\mu_{s}\right)
\end{array}\right)
$$

where $\mu_{b}=m_{b} \Sigma V, \mu_{v}=m_{v} \Sigma V$, and $\mu_{s}=m_{s} \Sigma V . K_{\nu}$ 's and $I_{\nu}$ 's are the modified Bessel functions. Note that, in the limit $\mu_{b} \rightarrow \mu_{v}$, (3) reduces to the zero-mode partition function of the full $N_{f}=2$ theory:

$$
\lim _{\mu_{b} \rightarrow \mu_{v}} Z_{\nu}^{\mathrm{PQ}}\left(\mu_{b} \mid \mu_{v}, \mu_{s}\right)=Z_{\nu}^{\text {full }}\left(\mu_{s}\right) \equiv \operatorname{det}\left(\begin{array}{cc}
I_{\nu}\left(\mu_{s}\right) & I_{\nu-1}\left(\mu_{s}\right) / \mu_{s} \\
\mu_{s} I_{\nu+1}\left(\mu_{s}\right) & I_{\nu}\left(\mu_{s}\right)
\end{array}\right) .
$$

Then the partially quenched chiral condensate at finite $V$ and $\nu$ is given by 


$$
\begin{aligned}
\frac{\sum_{\nu}^{\mathrm{PQ}}\left(\mu_{v}, \mu_{s}\right)}{\Sigma} & \equiv-\lim _{\mu_{b} \rightarrow \mu_{v}} \frac{\partial}{\partial \mu_{b}} \ln Z_{\nu}^{\mathrm{PQ}}\left(\mu_{b} \mid \mu_{v}, \mu_{s}\right) \\
& =\frac{-1}{Z_{\nu}^{\text {full }}\left(\mu_{s}\right)\left(\mu_{s}^{2}-\mu_{v}^{2}\right)^{2}} \operatorname{det}\left(\begin{array}{cccc}
\partial_{\mu_{v}} K_{\nu}\left(\mu_{v}\right) & I_{\nu}\left(\mu_{v}\right) & I_{\nu}\left(\mu_{s}\right) & I_{\nu-1}\left(\mu_{s}\right) / \mu_{s} \\
-\partial_{\mu_{v}}\left(\mu_{v} K_{\nu+1}\left(\mu_{v}\right)\right) & \mu_{v} I_{\nu+1}\left(\mu_{v}\right) & \mu_{s} I_{\nu+1}\left(\mu_{s}\right) & I_{\nu}\left(\mu_{s}\right) \\
\partial_{\mu_{v}}\left(\mu_{v}^{2} K_{\nu+2}\left(\mu_{v}\right)\right) & \mu_{v}^{2} I_{\nu+2}\left(\mu_{v}\right) & \mu_{s}^{2} I_{\nu+2}\left(\mu_{s}\right) & \mu_{s} I_{\nu+1}\left(\mu_{s}\right) \\
-\partial_{\mu_{v}}\left(\mu_{v}^{3} K_{\nu+3}\left(\mu_{v}\right)\right) & \mu_{v}^{3} I_{\nu+3}\left(\mu_{v}\right) & \mu_{s}^{3} I_{\nu+3}\left(\mu_{s}\right) & \mu_{s}^{2} I_{\nu+2}\left(\mu_{s}\right)
\end{array}\right) .
\end{aligned}
$$

It is not difficult to see that in the $\mu_{v} \rightarrow \mu_{s}$ limit, the partially quenched condensate reduces to the one in the full theory,

$$
\frac{\sum_{\nu}^{\mathrm{PQ}}\left(\mu_{s}, \mu_{s}\right)}{\Sigma}=\frac{\sum_{\nu}^{\mathrm{full}}\left(\mu_{s}\right)}{\Sigma} \equiv \frac{1}{2} \frac{\partial}{\partial \mu_{s}} \ln Z_{\nu}^{\text {full }}\left(\mu_{s}\right) .
$$

In the following, we will also use a second-derivative

$$
\frac{\Delta \Sigma_{\nu}^{\mathrm{PQ}}\left(\mu_{v}, \mu_{s}\right)}{\Sigma} \equiv \frac{\lim _{\mu_{b} \rightarrow \mu_{v}} \partial_{\mu_{b}} \partial_{\mu_{v}} Z_{\nu}^{\mathrm{PQ}}\left(\mu_{b} \mid \mu_{v}, \mu_{s}\right)}{Z_{\nu}^{\text {full }}\left(\mu_{s}\right)} .
$$

First we present the two-point correlation functions of the flavored pseudoscalar and scalar operators, $P^{a}(x)=$ $\bar{q}(x) \tau^{a} \gamma_{5} q(x)$ and $S^{a}(x)=\bar{q}(x) \tau^{a} q(x)$, where $\tau^{a}$ denotes a generator of $S U\left(N_{v}\right)$ group that the valence quark field $q(x)$ belongs to.

The expressions for the correlators $\left\langle P^{a}(x) P^{a}(0)\right\rangle$ and $\left\langle S^{a}(x) S^{a}(0)\right\rangle$ in the partially quenched ChPT is known to $\mathcal{O}\left(\epsilon^{2}\right)$ (no sum over $a$ ) [41]:

$$
\begin{aligned}
C_{P}(t) \equiv & \int d^{3} x\left\langle P^{a}(x) P^{a}(0)\right\rangle \\
= & \frac{1}{2} \frac{L^{3} \sum_{\text {eff }}^{2}}{\mu_{v}^{\text {eff }}} \frac{\sum_{\nu}^{\mathrm{PQ}}\left(\mu_{v}^{\text {eff }}, \mu_{s}^{\text {eff }}\right)}{\Sigma} \\
& -\frac{1}{2}\left[\frac{2 \Sigma^{2}}{F^{2}} \frac{\Delta \Sigma_{\nu}^{\mathrm{PQ}}\left(\mu_{v}, \mu_{s}\right)}{\Sigma}+\frac{\Sigma^{2}}{F^{2}} \frac{\partial_{\mu_{v}} \Sigma_{\nu}^{\mathrm{PQ}}\left(\mu_{v}, \mu_{s}\right)}{\Sigma}\right. \\
& -\frac{\Sigma^{2}}{F^{2}} \frac{4}{\mu_{v}^{2}-\mu_{s}^{2}}\left(\frac{\mu_{v} \Sigma_{\nu}^{\mathrm{PQ}}\left(\mu_{v}, \mu_{s}\right)}{\Sigma}\right. \\
& \left.\left.-\frac{\mu_{s} \Sigma_{\nu}^{\text {full }}\left(\mu_{s}\right)}{\Sigma}\right)\right] T h_{1}(t / T),
\end{aligned}
$$

$$
\begin{aligned}
C_{S}(t) \equiv & \int d^{3} x\left\langle S^{a}(x) S^{a}(0)\right\rangle \\
= & \frac{L^{3} \Sigma_{\text {eff }}^{2}}{2} \frac{\partial_{\mu_{v}} \Sigma_{\nu}^{\mathrm{PQ}}\left(\mu_{v}^{\mathrm{eff}}, \mu_{s}^{\mathrm{eff}}\right)}{\Sigma}-\frac{1}{2}\left[\frac{2 \Sigma^{2}}{F^{2}} \frac{\nu^{2}}{\mu_{v}^{2}}+\frac{\Sigma^{2}}{F^{2}}\right. \\
& \times \frac{1}{\mu_{v}} \frac{\sum_{\nu}^{\mathrm{PQ}}\left(\mu_{v}, \mu_{s}\right)}{\Sigma}-\frac{\Sigma^{2}}{F^{2}} \frac{4}{\mu_{v}^{2}-\mu_{s}^{2}} \\
& \left.\times\left(\frac{\mu_{v} \Sigma_{\nu}^{\mathrm{PQ}}\left(\mu_{v}, \mu_{s}\right)}{\Sigma}-\frac{\mu_{s} \Sigma_{\nu}^{\mathrm{full}}\left(\mu_{s}\right)}{\Sigma}\right)\right] T h_{1}(t / T) .
\end{aligned}
$$

The operators are separated in time by $t$ and projected onto zero spatial momentum. At the NLO an "effective" chiral condensate $\Sigma_{\text {eff }}$,

$$
\Sigma_{\mathrm{eff}}=\Sigma\left(1+\frac{3 \beta_{1}}{2 F^{2} V^{1 / 2}}\right)
$$

appears with a combination $\mu_{i}^{\text {eff }}=m_{i} \Sigma_{\text {eff }} V$. The parameter $\beta_{1}$ is the so-called shape coefficient that depends on the shape of the box. In our numerical study, $\beta_{1}=0.0836$. In Eqs. (8) and (9), the time dependence is simply written by a quadratic function $h_{1}(t / T)$ as

$$
h_{1}(t / T) \equiv \frac{1}{2}\left[\left(\frac{t}{T}-\frac{1}{2}\right)^{2}-\frac{1}{12}\right] .
$$

Next, we consider the flavored axial-vector and vector operators $A_{0}^{a}(x)=\bar{q}(x) \tau^{a} \gamma_{0} \gamma_{5} q(x) \quad$ and $\quad V_{0}^{a}(x)=$ $\bar{q}(x) \tau^{a} \gamma_{0} q(x)$. These correlators to $\mathcal{O}\left(\epsilon^{2}\right)$ in the $N_{f}=2$ unquenched ChPT are [39]

$$
\begin{aligned}
C_{A}(t) \equiv & \int d^{3} x\left\langle A_{0}^{a}(x) A_{0}^{a}(0)\right\rangle \\
= & -\frac{F^{2}}{2 T}\left\{\mathcal{J}_{+}^{0}+\frac{2}{F^{2}}\left(\frac{\beta_{1}}{V^{1 / 2}} \mathcal{J}_{+}^{0}-\frac{T^{2}}{V} k_{00} \mathcal{J}_{-}^{0}\right)\right. \\
& \left.+\frac{4 \mu_{s}}{F^{2}} \frac{\Sigma_{\nu}^{\text {full }}\left(\mu_{s}\right)}{\Sigma} \frac{T^{2}}{V} h_{1}(t / T)\right\}, \\
C_{V}(t) \equiv & \int d^{3} x\left\langle V_{0}^{a}(x) V_{0}^{a}(0)\right\rangle \\
= & -\frac{F^{2}}{2 T}\left\{\mathcal{J}_{-}^{0}+\frac{2}{F^{2}}\left(\frac{\beta_{1}}{V^{1 / 2}} \mathcal{J}_{-}^{0}-\frac{T^{2}}{V} k_{00} \mathcal{J}_{+}^{0}\right)\right\},
\end{aligned}
$$

where $k_{00}$ is another numerical factor depending on the shape of the box. In our numerical study, $k_{00}=0.08331$. $\mathcal{J}_{ \pm}^{0}$ 's are defined by

$$
\begin{aligned}
\mathcal{J}_{+}^{0} \equiv & \frac{1}{3}\left(2+2\left[\frac{\partial_{\mu_{s}} \Sigma_{\nu}^{\text {full }}\left(\mu_{s}^{\text {eff }}\right)}{\Sigma}+2\left(\frac{\sum_{\nu}^{\text {full }}\left(\mu_{s}^{\text {eff }}\right)}{\Sigma}\right)^{2}\right.\right. \\
& \left.\left.+\frac{1}{\mu_{s}^{\text {eff }}} \frac{\sum_{\nu}^{\text {full }}\left(\mu_{s}^{\text {eff }}\right)}{\Sigma}-2 \frac{\nu^{2}}{\left(\mu_{s}^{\text {eff }}\right)^{2}}\right]\right), \\
\mathcal{J}_{-}^{0} \equiv & \frac{1}{3}\left(4-2\left[\frac{\partial_{\mu_{s}} \Sigma_{\nu}^{\text {full }}\left(\mu_{s}^{\text {eff }}\right)}{\Sigma}+2\left(\frac{\sum_{\nu}^{\text {full }}\left(\mu_{s}^{\text {eff }}\right)}{\Sigma}\right)^{2}\right.\right. \\
& \left.\left.+\frac{1}{\mu_{s}^{\text {eff }}} \frac{\sum_{\nu}^{\text {full }}\left(\mu_{s}^{\text {eff }}\right)}{\Sigma}-2 \frac{\nu^{2}}{\left(\mu_{s}^{\text {eff }}\right)^{2}}\right]\right) .
\end{aligned}
$$

It should be noted that the axial-vector and vector correlators are sensitive to $F$, as it appears as an overall constant. Their time dependence and other factors repre- 
sent the NLO effect. For the pseudoscalar and scalar correlators, on the other hand, $\Sigma$ determines the overall constant and $F$ appears only in the NLO correction term. Therefore, by using both types of correlators the two LECs can be extracted with a good sensitivity.

\section{LATTICE SIMULATIONS}

We summarize the setup of our numerical simulations. Details of the configuration generation and the eigenvalue calculations are given in [22].

Our lattice is $16^{3} \times 32$ at a lattice spacing $a=$ $0.1111(24) \mathrm{fm}$ determined from the Sommer scale $r_{0}=$ $0.49 \mathrm{fm}$ as an input [32]. We employ the overlap fermion $[17,18]$, defined by the Dirac operator

$$
D(m)=\left(m_{0}+\frac{m}{2}\right)+\left(m_{0}-\frac{m}{2}\right) \gamma_{5} \operatorname{sgn}\left[H_{W}\left(-m_{0}\right)\right]
$$

for a quark mass $m . H_{W}\left(-m_{0}\right) \equiv \gamma_{5} D_{W}\left(-m_{0}\right)$ denotes the standard Hermitian Wilson-Dirac operator at a large negative mass $-m_{0}$. We choose $m_{0}=1.6$ throughout this work. We remark that the mass parameters are given in the lattice unit unless otherwise stated. We employ the Iwasaki action $[33,34]$ for the gauge field at $\beta=2.35$ with an additional determinant factor $\operatorname{det}\left[H_{W}^{2} /\left(H_{W}^{2}+m_{t}^{2}\right)\right]$ in the partition function produced by extra Wilson fermions and twistedmass ghosts [35-37] (we set $m_{t}=0.2$ ). With this choice the global topological charge does not change its value during the molecular dynamics updates of the Hybrid Monte Carlo algorithm. Fixing topology in this way is desirable for the study of the $\epsilon$-regime of QCD, since the analytical expressions of ChPT are given at a fixed topological sector. In this work, we take the trivial topological sector $\nu=0$ only.

The sign function in (16) is approximated by a rational function with Zolotarev's coefficients after projecting out a few lowest eigenmode's contribution. With 10 poles the accuracy of the sign function is $10^{-(7-8)}$. Therefore, the violation of the chiral symmetry due to the lattice action is negligible in our work.

In this work, we use the gauge configurations generated at sea quark mass $m=0.002$, which corresponds to $\sim 3 \mathrm{MeV}$. For this value the parameter $m \Sigma V$ is about 0.556 , and the system is well within the $\epsilon$-regime. As we decrease the quark mass from the $p$-regime $(m \Sigma V \gg 1)$ to the $\epsilon$-regime $(m \Sigma V \lesssim 1)$, the lowest eigenvalue of $D(m)^{\dagger} D(m), \simeq \lambda_{1}^{2}+m^{2}$, is bounded from below by a repulsion of the lowest eigenvalue from zero due to the fermion determinant $\prod_{k}\left(\lambda_{k}^{2}+m^{2}\right)$. The condition number of the operator $D(m)^{\dagger} D(m)$, and thus the computational cost for its inversion, saturates near the boarder between the $p$-regime and the $\epsilon$-regime. As a consequence, for example, the number of multiplication of the WilsonDirac operator $D_{W}$ needed per trajectory at $m=0.002$ is only about 1.5 times greater than that at 10 times heavier sea quark mass [22]. We have accumulated 4600 HMC trajectories after discarding 400 trajectories for thermalization. The numerical cost is about one hour per trajectory on a half rack (512 nodes) of the IBM BlueGene/L (2.8 TFlops peak performance).

At every 10 trajectories, we calculate the meson correlators at four values of valence quark masses $m=0.0005$, $0.0010,0.0020$, and 0.0030 ranging $1-4 \mathrm{MeV}$ in the physical unit. For the inversion of the overlap-Dirac operator we use the multishift conjugate gradient (CG) solver to calculate all the valence quark propagators simultaneously. The solver is accelerated by projecting out the subspace spanned by 50 pairs of lowest-lying eigenmodes. [The eigenvalues of $D(0)$ form a pair with their complex conjugate; the eigenvector of the counterpart is produced by multiplying $\gamma_{5}$.] With this projection, the solver performance is an order of magnitude better and roughly independent of the quark mass. These eigenmodes of $D(0)$ have been calculated using the implicitly restarted Lanczos algorithm and stored on disks for studying the eigenvalue distribution [21,22].

We compute the meson correlators with the low-mode averaging (LMA) technique [13,45]. Using the $N_{e p}$ pairs of lowest-lying eigenmodes, we decompose the quark propagator $D(m)^{-1}$ into the low-mode contribution $[D(m)]_{\text {low }}^{-1}$ and the rest $[D(m)]_{\text {high }}^{-1}$ as

$$
\begin{aligned}
D(m)^{-1}(x, y)= & {[D(m)]_{\text {low }}^{-1}(x, y)+[D(m)]_{\mathrm{high}}^{-1}(x, y) } \\
= & \sum_{k=1}^{N_{e p}}\left[\frac{u_{k}(x) u_{k}^{\dagger}(0)}{\left(1-m / 2 m_{0}\right) \lambda_{k}+m}\right. \\
& \left.+\frac{\gamma_{5} u_{k}(x) u_{k}^{\dagger}(0) \gamma_{5}}{\left(1-m / 2 m_{0}\right) \lambda_{k}^{*}+m}\right] \\
& +[D(m)]_{\text {high }}^{-1}(x, y)
\end{aligned}
$$

where $u_{k}(x)$ is the eigenvector of $D(0)$ associated with its eigenvalue $\lambda_{k}$. While the high-mode contribution has to be obtained by using the CG solver for a fixed source point $y$, the low-mode contribution can be calculated from the $50+$ 50 low-lying modes for any source and sink points without extra cost. With the LMA technique, we average over the source point for a part of the meson correlator that is purely composed of $[D(m)]_{\text {low }}^{-1}$. For other contributions, we simply use a fixed source $y$ at the origin.

The LMA technique is effective to improve the statistical signal when the correlator of interest is dominated by the low-mode contribution. In Fig. 1 we demonstrate the improvement by taking pseudoscalar and scalar correlators calculated on a single gauge configuration as an example. Some wiggle observed without LMA is completely washed out with LMA, and smooth curve is obtained. A similar comparison is shown in Fig. 2 for the axial-vector and vector correlators, for which the improvement with LMA is marginal. This indicates that these correlators are not simply dominated by the low-lying modes. Note that the 

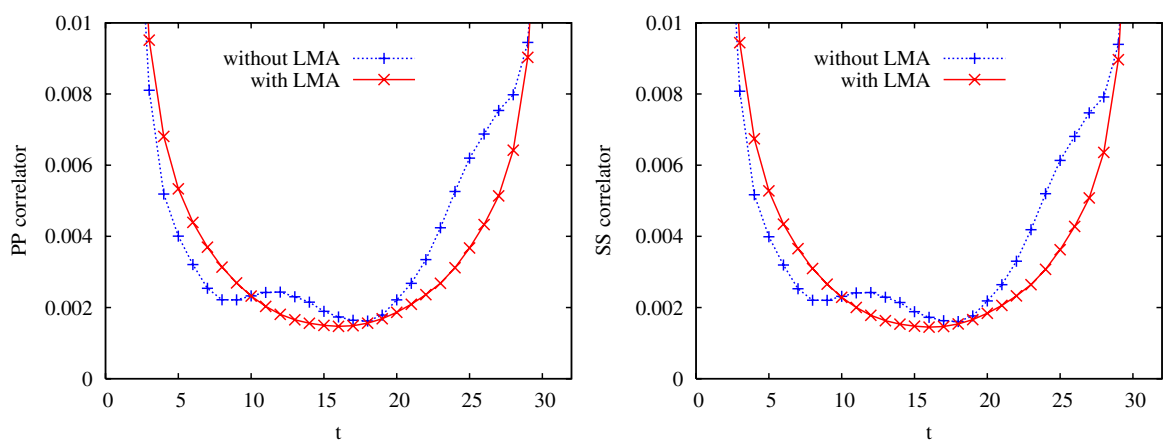

FIG. 1 (color online). Pseudoscalar (left) and scalar (right) correlators with (solid curve) and without (dotted curve) the low-mode averaging. Correlators are calculated on a single gauge configuration at $m=0.002$.
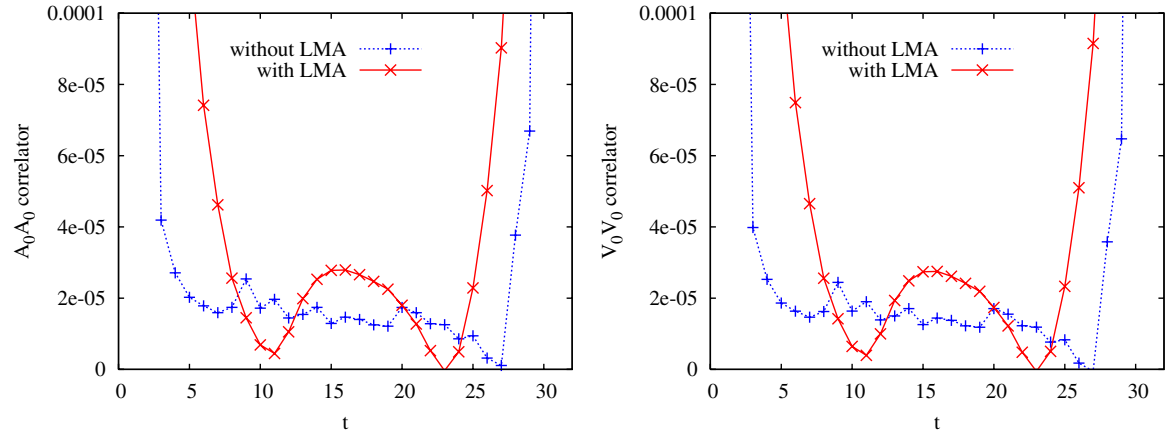

FIG. 2 (color online). Axial-vector (left) and vector (right) correlators with (solid curve) and without (dotted curve) the low-mode averaging. Correlators are calculated on a single gauge configuration at $m=0.002$.

magnitude of these correlators is 2 orders of magnitude smaller than pseudoscalar and scalar correlators.

For the statistical analysis, we use the jackknife method with a bin size 20, which corresponds to 200 HMC trajectories. With this choice the statistical error saturates for the calculation of the averaged low-lying eigenvalues $\left\langle\lambda_{k}\right\rangle$ as
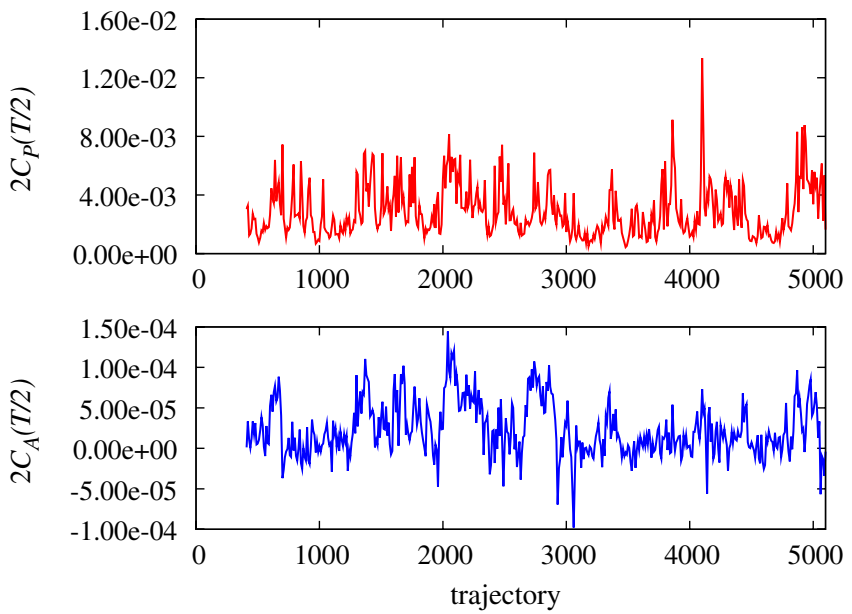

FIG. 3 (color online). The Monte Carlo history of the pseudoscalar correlator (top) and the axial-vector correlator (bottom) at $t=T / 2=16$. studied in [22]. Since the low-lying modes reflect the longdistance physics (zero modes of pion fields in the language of (hPT), these quantities are expected to have the longest autocorrelation time among other physical quantities. In Fig. 3, we plot the Monte Carlo history of the quantities of interest, i.e. the pseudoscalar and axial-vector correlators at the largest time separation $t=T / 2=16, C_{P}(T / 2)$, and $C_{A}(T / 2)$ (see the definition given later). We observe that the 200-trajectory gives a reasonable range of the autocorrelation. However, the integrated autocorrelation time calculated following the definition in [46] is substantially shorter: $37(12)$ and 47(16) HMC trajectories for $C_{P}(T / 2)$ and $C_{A}(T / 2)$, respectively. We therefore conclude that the binsize of 20 is a conservative choice.

\section{NUMERICAL RESULTS}

In this section we explain the fit of our data to the (partially quenched) ChPT formulas in Eqs. (8), (9), (12), and (13).

First, we study the axial-vector current correlator at $m_{v}=m_{s}=0.002$ (full QCD point). Since we use the local axial current $A_{0}^{a}(x)$, which is not a conserved current on the lattice, we need a finite renormalization to relate the lattice current to the continuum current $\mathcal{A}_{0}^{a}(x)$ as $\mathcal{A}_{0}^{a}(x)=$ $Z_{A} A_{0}^{a}(x)$. We calculated the renormalization factor $Z_{A}$ nonperturbatively through the axial Ward identity and obtain 
$Z_{A}=1.3513(13)$. In the following, the values quoted for $F$ include this $Z$-factor, neglecting its tiny statistical error.

Figure 4 shows the axial-vector current correlator with and without the LMA technique. Although the improvement by LMA is marginal for this channel, the statistical error is reduced by about $30 \%$. We fit the data to the ChPT formula (12) taking $F$ and $\Sigma$ as free parameters. With a fitting range $t \in[12,20]$ we obtain the solid curve shown in Fig. 4. Roughly speaking, the overall magnitude (the constant piece) determines $F$, while the curvature [or the term proportional to $h_{1}(t / T)$ ] gives $\Sigma$. We obtain $\Sigma \sim$ $[260(32) \mathrm{MeV}]^{3}$ and $F \sim 90(6) \mathrm{MeV}$. The statistical error is large for $\Sigma$ (about 30\%), because it is extracted from a tiny curvature.

A similar plot is obtained for the vector channel as shown in Fig. 5. At the leading order, i.e. in the massless limit at finite $V$, axial-vector and vector correlators become identical because of the exact chiral symmetry; the difference arises due to the zero-mode integrals when $\mu$ is finite. The line in the plot shows the formula (13) with the parameters $F$ and $\Sigma$ obtained from the fit of the axialvector and pseudoscalar channels (see Sec. V). It shows a remarkable consistency.

Next, we consider the pseudoscalar channel at $m_{v}=$ $m_{s}=0.002$. The lattice data obtained with the LMA technique are shown in Fig. 6 . With $F$ obtained via the axialvector correlator as an input, we compare the pseudoscalar channel with the chiral perturbation theory formula at the NLO (8). The motivation for this choice is that the pseudoscalar channel itself does not have a good sensitivity on $F$, as it appears only at the NLO. From a fit in the range $t \in[12,20]$ we obtain the chiral condensate as $\Sigma \sim$ $[227(4) \mathrm{MeV}]^{3}$.

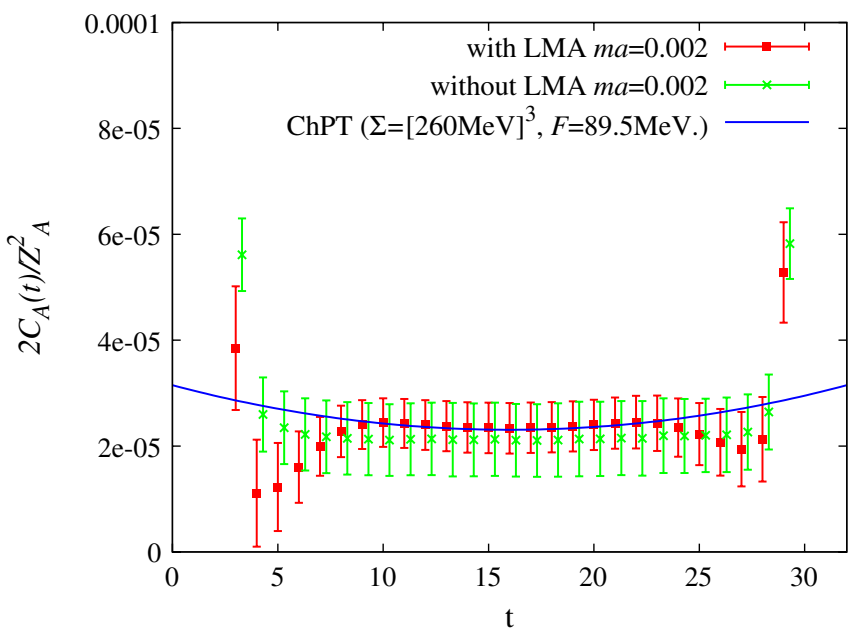

FIG. 4 (color online). Axial-vector correlator in the $\epsilon$-regime. Filled square symbols denote the low-mode averaged correlator while the crosses are not averaged. The solid curve shows the best fit with the ChPT formula.

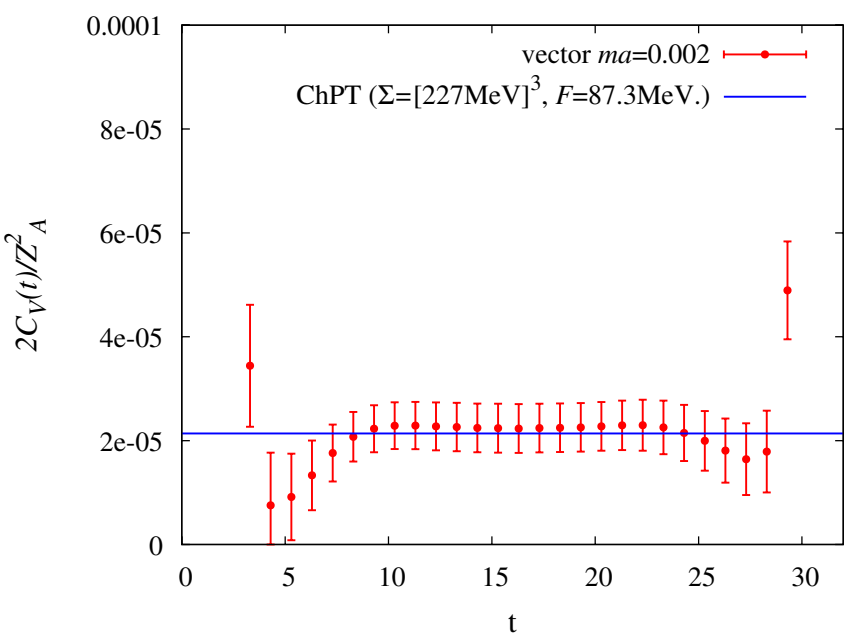

FIG. 5 (color online). Vector correlator in the $\epsilon$-regime. The solid curve is the ChPT prediction with inputs $\Sigma=$ $\left[227.6(3.7) \mathrm{MeV}^{3}\right.$ and $F=87.3(5.6) \mathrm{MeV}$ (no free parameter left).

A similar result can be obtained for the scalar channel as plotted in Fig. 7. We draw a curve representing the ChPT formula (9) with the parameters obtained from the fit of the axial-vector and pseudoscalar channels (see Sec. V). Again for this channel, the data are consistent with the expectation within the statistical error.

Using the data at $m_{v} \neq m_{s}$ we can check the consistency of the lattice results with the partially quenched ChPT formulas (8) and (9). Figure 8 shows the pseudoscalar and scalar correlators at four different valence quark masses ranging 1-4 MeV while fixing the sea quark mass at $3 \mathrm{MeV}$. The shape of the correlators does not strongly depend on the valence quark mass, but some dependence can be seen. With the input parameters $F$ and $\Sigma$ determined from a global fit of the axial-vector

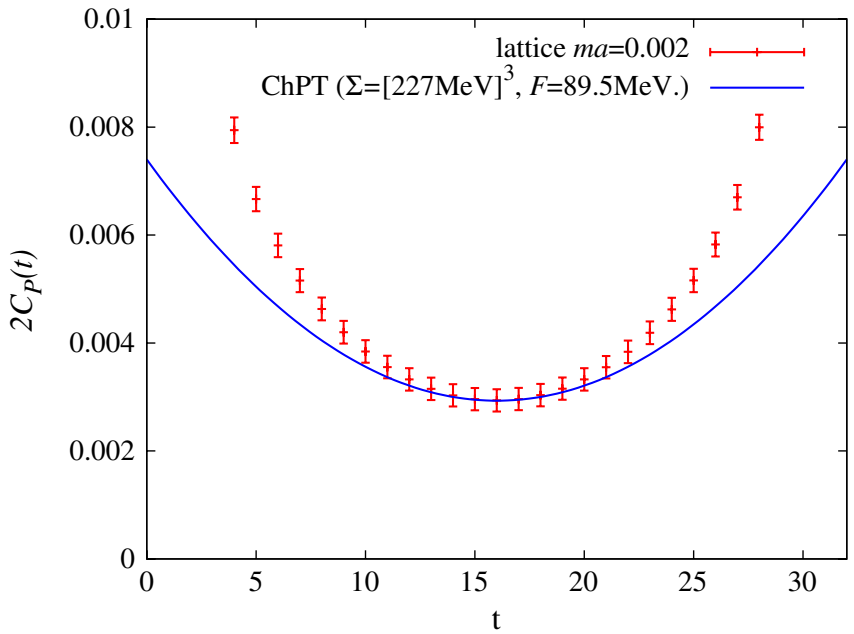

FIG. 6 (color online). Pseudoscalar correlator in the $\epsilon$-regime. The solid curve represents a fit with the NLO ChPT formula. 
LATTICE STUDY OF MESON CORRELATORS IN THE ...

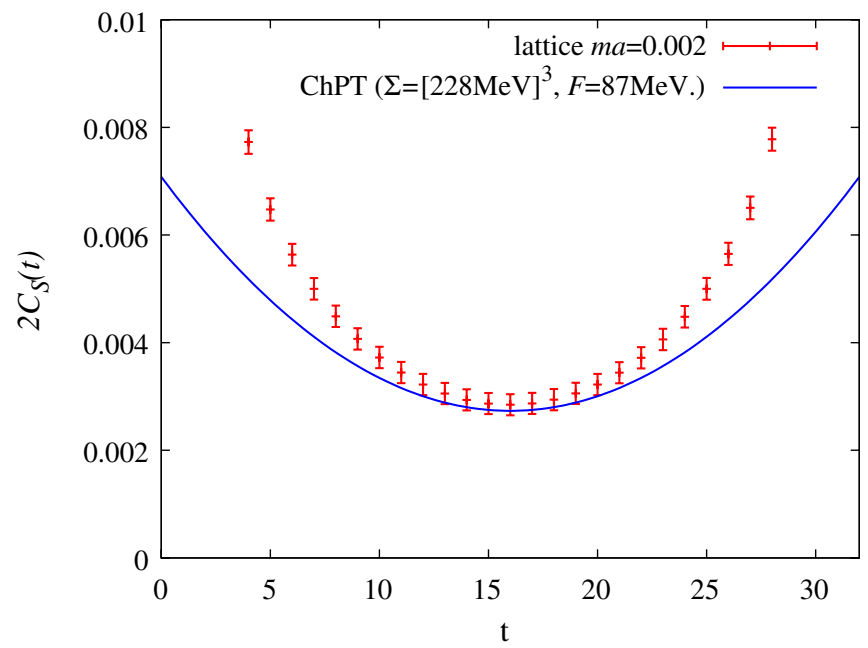

FIG. 7 (color online). Scalar correlator in the $\epsilon$-regime. The solid curve represents a fit with the NLO ChPT formula.
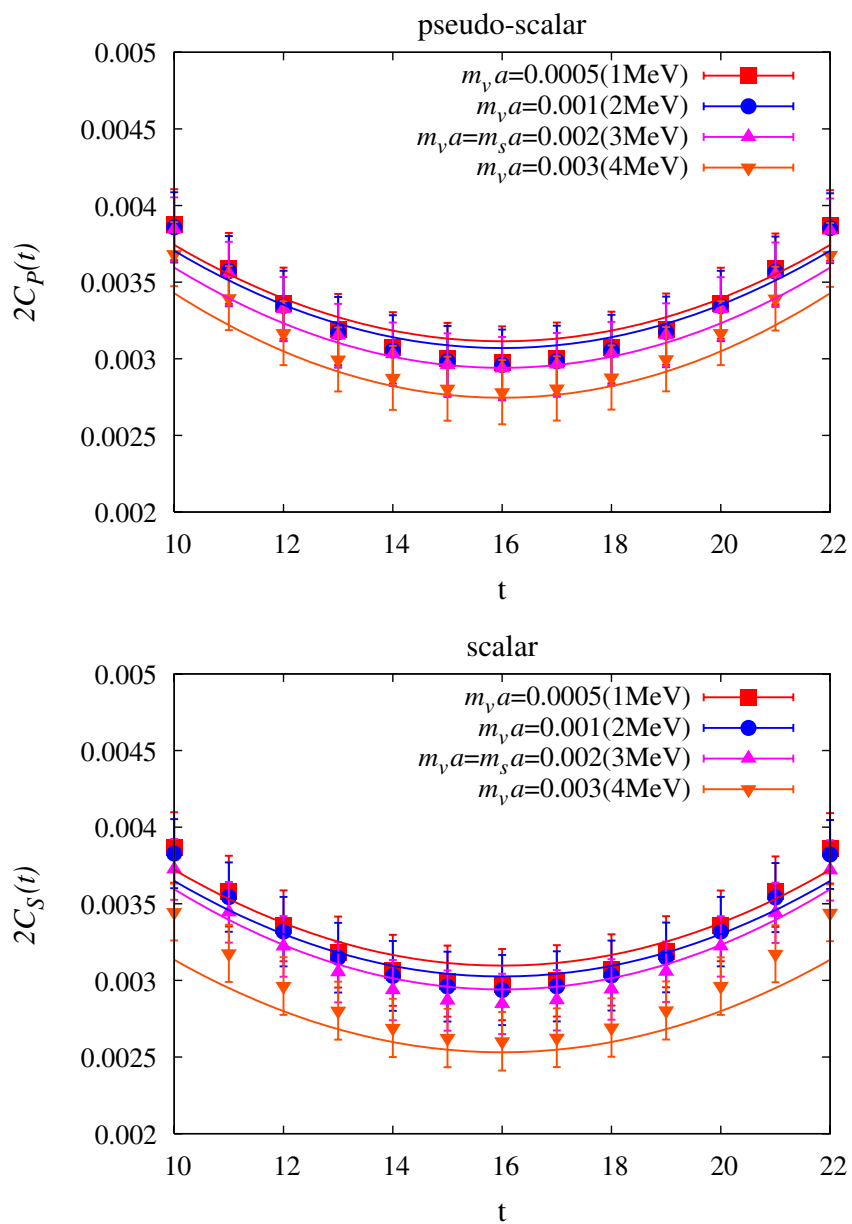

FIG. 8 (color online). Partially quenched pseudoscalar (top) and scalar (bottom) correlators in the $\epsilon$-regime. The solid curves are the ChPT results with $\Sigma=\left[227.6(3.7) \mathrm{MeV}^{3}\right.$ and $F=$ 87.3(5.6) $\mathrm{MeV}$ as inputs (no free parameter left).
PHYSICAL REVIEW D 77, 074503 (2008)
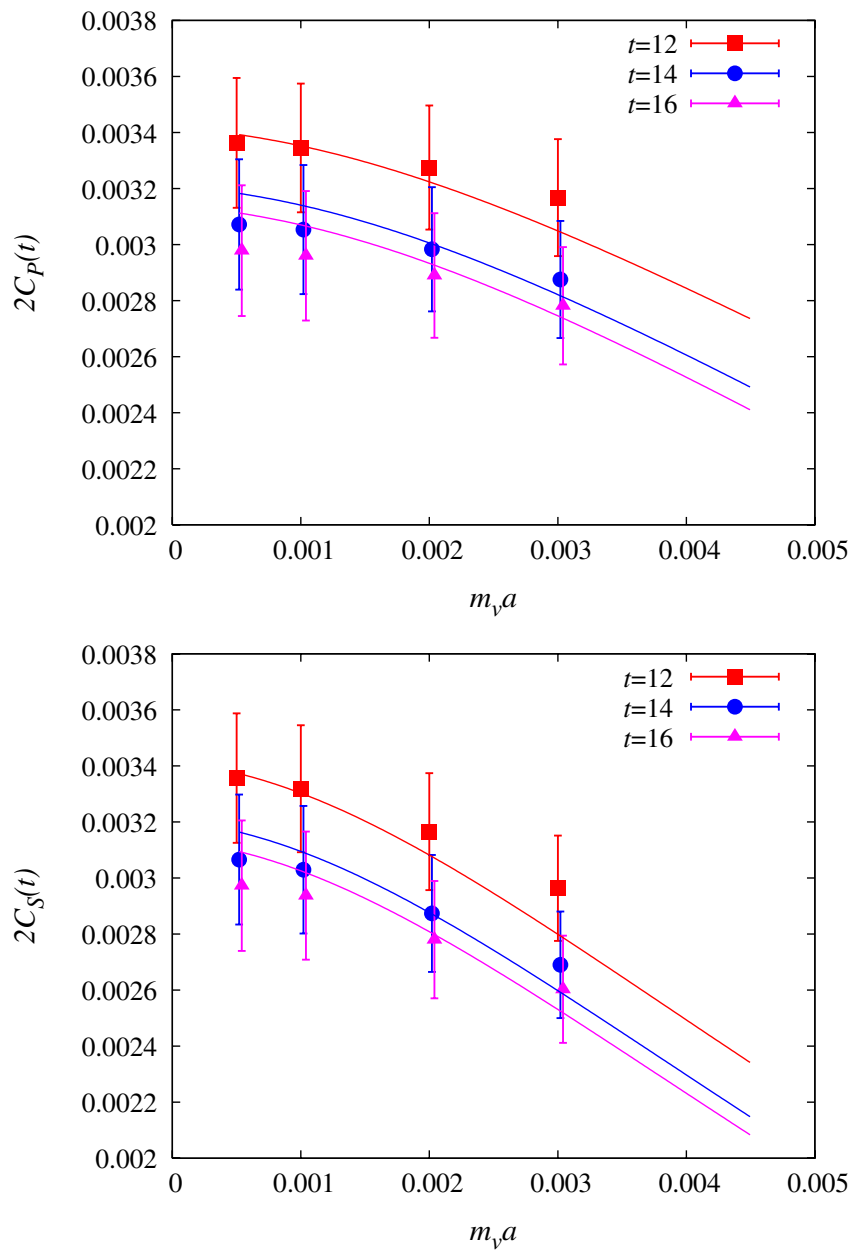

FIG. 9 (color online). Valence quark mass dependence of the partially quenched pseudoscalar (top) and scalar (bottom) correlators in the $\epsilon$-regime at each time slice.

and pseudoscalar channel (see Sec. V), we draw the expectation from the partially quenched ChPT in Fig. 8. There is no additional parameters in this analysis, hence it gives a stringent test of either the NLO ChPT formula or the lattice calculation. We find an excellent agreement for all the valence quark masses within the statistical error. Some specific points of the correlator, e.g. at $t=12,14$, or 16 , are plotted as a function of the valence quark mass in Fig. 9. The decrease of the correlators with the valence quark mass is nicely reproduced by the lattice data.

\section{EXTRACTION OF THE LOW-ENERGY CONSTANTS}

Since the axial-vector and pseudoscalar channels are complementary to each other in determining the LECs $F$ and $\Sigma$, we fit both channels simultaneously with these two parameters to obtain our best results: $\Sigma=$ $[227.6(3.7) \mathrm{MeV}]^{3}$ and $F=87.3(5.6) \mathrm{MeV}$. Here, the statistical error of the lattice spacing $a$ is also taken into account. The fit range is again $t \in[12,20]$ for both chan- 
nels. Under the change of the lower limit of the fitting range from 10 to 15 , which corresponds to 1.1 to $1.7 \mathrm{fm}$, the fit results are quite stable (within 1\%) with similar error bars.

Multiplying the nonperturbative renormalization factor [47] to convert our result of the chiral condensate to the continuum $\overline{\mathrm{MS}}$ scheme we obtain $\sum^{\overline{\mathrm{MS}}}(2 \mathrm{GeV})=$ $\left[239.8(4.0) \mathrm{MeV}^{3}\right.$, where the error represents the statistical one.

Possible sources of the systematic error include the discretization effect of $O\left(a^{2}\right)$ and the higher order effect of the $\epsilon$-expansion. Since we do not have data at different lattice spacings, we cannot quantify the discretization effect beyond a rough order counting. Assuming that the relevant physical scale is the QCD scale $\Lambda_{\mathrm{QCD}} \sim$ $300-500 \mathrm{MeV}$, the size of $\mathcal{O}\left(\left(a \Lambda_{\mathrm{QCD}}\right)^{2}\right)$ effect is $3 \%-$ $8 \%$. This small scaling violation is supported for the overlap fermions by recent quenched simulations (in the $p$-regime) $[48,49]$, albeit for different physical quantities. The unphysical (heavy) Wilson fermions we introduced to fix the topological charge should not have any negative impact on the scaling, because they never arise in the external states and merely affect the gluon action at order $a^{2}$ in the Symanzik's effective theory. Their effect on the effective gluon action is further minimized by the accompanied ghosts that cancel the Wilson fermion contributions except for the near-zero modes of $H_{W}$, that appear for locally bumpy gauge configurations, or for the so-called dislocations [50].

The higher order effect of the $\epsilon$-expansion appears due to the finite volume lattice. In our analysis, the NLO terms, i.e. $\mathcal{O}\left(\epsilon^{2}\right)$ terms, are included and the remaining corrections are of $\mathcal{O}\left(\epsilon^{4}\right) \sim \mathcal{O}\left(1 /\left(\Lambda_{\text {cut }} L\right)^{4}\right)$, with $\Lambda_{\text {cut }}$ the cutoff scale of ChPT. With a conservative choice $\Lambda_{\text {cut }} \sim$ $300-500 \mathrm{MeV}$, this uncertainty is $0.3 \%-2 \%$. This order counting gives a reasonable estimate for the NLO correction to $\Sigma$. Namely, the size of the NLO correction $\left(\Sigma_{\text {eff }}-\right.$ $\Sigma) / \Sigma$ [see (10)] estimated with this order counting is $14 \%$, while the real correction was $20 \%$. This agreement indicates that the order-counting argument is indeed valid up to an $O(1)$ factor, which depends on the shape of the spacetime box [6] and could be sizable at the next-to-next-toleading order. At the same order of the $\epsilon$-expansion, there is a contribution from the finite pion mass $\mathcal{O}\left(\left(m_{\pi} / \Lambda_{\text {cut }}\right)^{2}\right)$, which numerically gives about $2 \%$. We note that such a small uncertainty does not apply for the curvature of the correlators, since the curvature itself is the quantity arising at the NLO. But the extraction of $F$ and $\Sigma$ relies mainly on the constant piece of the correlators, that starts from the leading order in the $\epsilon$-expansion.

Adding the uncertainties from the scaling violation and from the higher order effects in the $\epsilon$-expansion in quadrature we estimate the dominant systematic error is of order $9 \%$. For a more robust estimate beyond the order counting, it is important to study the finite lattice spacing and volume effects in the future works.
The result for the decay constant $F$ may be compared with the calculation in the $p$-regime [51]. In [52] a preliminary result for the pion decay constant with two-flavors of dynamical overlap fermion is presented. With the same lattice action, the calculation is done at slightly coarser lattice spacing, $a \simeq 0.12 \mathrm{fm}$. An analysis with the next-tonext-to-leading order ChPT yields $F=78.6(2.7) \mathrm{MeV}$ (the error denotes the statistical one), which is slightly lower than the calculation in this work. Assuming the 9\% systematic error, however, both results are consistent with each other.

The result for the chiral condensate $\Sigma$ may be compared with the extraction from the lowest-lying eigenvalue through the chiral random matrix theory [21,22]: $\Sigma^{\overline{\mathrm{MS}}}(2 \mathrm{GeV})=[251(7)(11) \mathrm{MeV}]^{3}$. Here, the errors represent the statistical and the systematic due to the NLO correction in the $\epsilon$-expansion. The calculation in this work from the meson correlators includes the NLO correction, and in fact the result deviates from the previous result by about the estimated NLO correction. When one integrates out the nonzero modes of the pion field from the chiral Lagrangian, there remains the zero-mode integrals with an effective chiral condensate, which is given as

$$
\Sigma_{\text {eff }}=\Sigma\left(1+\frac{3}{2} \frac{0.0836}{F^{2} L^{2}}\right)
$$

at the one-loop order. The one-loop correction is a substantial effect [53]: a factor of 1.202. If we correct the value in this work with this amount we obtain $[255(4) \mathrm{MeV}]^{3}$, which is now consistent with the previous result within the small statistical error. This remarkable consistency has already been discussed in [24,26-28], and an argument based on an analytical calculation has been given recently [54]. It is also notable that our results are consistent with the topological susceptibility, from which we extracted $\Sigma^{\overline{\mathrm{MS}}}(2 \mathrm{GeV})=[254(5)(10) \mathrm{MeV}]^{3}$ (see $[55,56]$ for the details).

\section{CONCLUSION}

The extraction of the low-energy constants $F$ and $\Sigma$ from the meson correlators in the $\epsilon$-regime has a significant advantage over the conventional approach. Already at the NLO in the $\epsilon$-expansion, the remaining higher order effect is a percent level, and thus a precise calculation of both $F$ and $\Sigma$ is possible without delicate chiral extrapolations.

The dynamical lattice simulation is feasible with a small sea quark mass $\sim 3 \mathrm{MeV}$ on a $16^{3} \times 32$ lattice with $L \sim$ $1.7 \mathrm{fm}$, where the scale is determined assuming $r_{0}=$ $0.49 \mathrm{fm}$. Meson correlators are obtained with a good precision by using the low-mode averaging technique. The numerical results passed all the consistency checks for different channels as well as for the partially quenched mass combinations. Using the NLO ChPT formulas in the 
$\epsilon$-expansion, we obtain $\quad \Sigma^{\overline{\mathrm{MS}}}(2 \mathrm{GeV})=$ $[239.8(4.0) \mathrm{MeV}]^{3}$ and $F=87.3(5.6) \mathrm{MeV}$ with a possible systematic error of order $9 \%$ which is dominated by the discretization error. Reducing the systematic error by repeating the calculation on a larger lattice at smaller lattice spacing will be an important future work.

\section{ACKNOWLEDGMENTS}

H. F. thanks F. Bernardoni, P. H. Damgaard, T. DeGrand, L. Giusti, A. Hasenfratz, P. Hasenfratz, P. Hernández, S. Necco, and K. Splittorff for fruitful discussions. The nu- merical simulations are performed on IBM System Blue Gene Solution at High Energy Accelerator Research Organization (KEK) under support of its Large Scale Simulation Program (No. 07-16), also in part on NEC SX-8 at YITP, Kyoto University. This work is supported in part by Nishina foundation (HF) and by the Grants-inAid for Scientific Research from the Ministry of Education, Culture, Sports, Science and Technology No. 17740171, No. 18034011, No. 18340075, No. 18740167, No. 18840045, No. 19540286, and No. 19740160).
[1] S. Aoki, Phys. Rev. D 68, 054508 (2003).

[2] J. Gasser and H. Leutwyler, Phys. Lett. B 184, 83 (1987).

[3] J. Gasser and H. Leutwyler, Phys. Lett. B 188, 477 (1987).

[4] H. Neuberger, Phys. Rev. Lett. 60, 889 (1988).

[5] F. C. Hansen, Nucl. Phys. B345, 685 (1990); F. C. Hansen and H. Leutwyler, Nucl. Phys. B350, 201 (1991).

[6] P. Hasenfratz and H. Leutwyler, Nucl. Phys. B343, 241 (1990).

[7] H. Leutwyler and A. Smilga, Phys. Rev. D 46, 5607 (1992).

[8] L. Giusti, C. Hoelbling, M. Luscher, and H. Wittig, Comput. Phys. Commun. 153, 31 (2003).

[9] L. Giusti, M. Luscher, P. Weisz, and H. Wittig, J. High Energy Phys. 11 (2003) 023.

[10] K. Ogawa and S. Hashimoto, Prog. Theor. Phys. 114, 609 (2005).

[11] W. Bietenholz, T. Chiarappa, K. Jansen, K. I. Nagai, and S. Shcheredin, J. High Energy Phys. 02 (2004) 023.

[12] L. Giusti, P. Hernandez, M. Laine, P. Weisz, and H. Wittig, J. High Energy Phys. 01 (2004) 003.

[13] L. Giusti, P. Hernandez, M. Laine, P. Weisz, and H. Wittig, J. High Energy Phys. 04 (2004) 013.

[14] H. Fukaya, S. Hashimoto, and K. Ogawa, Prog. Theor. Phys. 114, 451 (2005).

[15] W. Bietenholz and S. Shcheredin, Nucl. Phys. B754, 17 (2006).

[16] L. Giusti and S. Necco, J. High Energy Phys. 04 (2007) 090.

[17] H. Neuberger, Phys. Lett. B 417, 141 (1998).

[18] H. Neuberger, Phys. Lett. B 427, 353 (1998).

[19] P. H. Ginsparg and K. G. Wilson, Phys. Rev. D 25, 2649 (1982).

[20] M. Luscher, Phys. Lett. B 428, 342 (1998).

[21] H. Fukaya et al. (JLQCD Collaboration), Phys. Rev. Lett. 98, 172001 (2007).

[22] H. Fukaya et al., Phys. Rev. D 76, 054503 (2007).

[23] H. Fukaya et al. (JLQCD Collaboration), Proc. Sci. (2007) 073.

[24] T. DeGrand, Z. Liu, and S. Schaefer, Phys. Rev. D 74, 094504 (2006); 74, 099904(E) (2006).

[25] C. B. Lang, P. Majumdar, and W. Ortner, Phys. Lett. B 649, 225 (2007)
[26] P. Hasenfratz, D. Hierl, V. Maillart, F. Niedermayer, A. Schafer, C. Weiermann, and M. Weingart, arXiv:0707.0071.

[27] T. DeGrand and S. Schaefer, Phys. Rev. D 76, 094509 (2007).

[28] T. DeGrand and S. Schaefer, arXiv:0709.2889.

[29] M. Joergler and C. B. Lang, Proc. Sci. LAT2007 (2007) 107.

[30] P. Hasenfratz, D. Hierl, V. Maillart, F. Niedermayer, A. Schafer, C. Weiermann, and M. Weingart, Proc. Sci. LAT2007 (2007) 077.

[31] K. Jansen, A. Nube, A. Shindler, C. Urbach, and U. Wenger, arXiv:0711.1871.

[32] R. Sommer, Nucl. Phys. B411, 839 (1994).

[33] Y. Iwasaki, Nucl. Phys. B258, 141 (1985).

[34] Y. Iwasaki and T. Yoshie, Phys. Lett. 143B, 449 (1984).

[35] T. Izubuchi and C. Dawson (RBC Collaboration), Nucl. Phys. B, Proc. Suppl. 106, 748 (2002).

[36] P. M. Vranas, Phys. Rev. D 74, 034512 (2006).

[37] H. Fukaya, S. Hashimoto, K. I. Ishikawa, T. Kaneko, H. Matsufuru, T. Onogi, and N. Yamada (JLQCD Collaboration), Phys. Rev. D 74, 094505 (2006).

[38] P. H. Damgaard, M. C. Diamantini, P. Hernandez, and K. Jansen, Nucl. Phys. B629, 445 (2002).

[39] P. H. Damgaard, P. Hernandez, K. Jansen, M. Laine, and L. Lellouch, Nucl. Phys. B656, 226 (2003).

[40] P. H. Damgaard and K. Splittorff, Phys. Rev. D 62, 054509 (2000).

[41] P. H. Damgaard and H. Fukaya, Nucl. Phys. B793, 160 (2008).

[42] F. Bernardoni and P. Hernandez, J. High Energy Phys. 10 (2007) 033.

[43] K. Splittorff and J. J. M. Verbaarschot, Phys. Rev. Lett. 90, 041601 (2003).

[44] Y. V. Fyodorov and G. Akemann, Pis'ma Zh. Eksp. Teor. Fiz. 77, 513 (2003) [JETP Lett. 77, 438 (2003)].

[45] T. A. DeGrand and S. Schaefer, Comput. Phys. Commun. 159, 185 (2004).

[46] M. Luscher, Comput. Phys. Commun. 165, 199 (2005).

[47] G. Martinelli, C. Pittori, C. T. Sachrajda, M. Testa, and A. Vladikas, Nucl. Phys. B445, 81 (1995).

[48] R. Babich, N. Garron, C. Hoelbling, J. Howard, L. 
Lellouch, and C. Rebbi, Phys. Rev. D 74, 073009 (2006).

[49] T. Draper et al., arXiv:hep-lat/0609034.

[50] M. Golterman and Y. Shamir, Phys. Rev. D 68, 074501 (2003).

[51] S. Necco, arXiv:0710.2444.

[52] J. Noaki et al. (JLQCD Collaboration), Proc. Sci. LAT2007 (2007) 126.

[53] P. H. Damgaard, T. DeGrand, and H. Fukaya, J. High
Energy Phys. 12 (2007) 060.

[54] F. Basile and G. Akemann, J. High Energy Phys. 12 (2007) 043.

[55] S. Aoki, H. Fukaya, S. Hashimoto, and T. Onogi, Phys. Rev. D 76, 054508 (2007).

[56] S. Aoki et al. (JLQCD and TWQCD Collaboration), arXiv:0710.1130. 